Pacific Journal of Mathematics

A NOTE ON MESOCOMPACT AND SEQUENTIALLy 


\title{
A NOTE ON MESOCOMPACT AND SEQUENTIALLY MESOCOMPACT SPACES
}

\author{
JAMES R. BOONE
}

\begin{abstract}
Characterizations of paracompactness in $k$-spaces have been obtained which employ the notions of mesocompact and sequentially mesocompact spaces. Property $(\omega)$ has been used to characterize collectionwise normality in sequential spaces, and applied to the study of metrizability of developable spaces.

It is the purpose of this paper present mapping theorems, in $\S 3$, which establish the invariance properties of normal mesocompact spaces, under perfect mappings, and normal sequentially mesocompact spaces, under closed presequential mappings. For this purpose, characterizations of these structures are developed in $\$ 2$, and the notion of a presequential mapping is introduced in $\S 3$. These characterizations are obtained by the use of two generalizations of collectionwise normality, property $(k)$ and property $(\omega)$, which are defined and studied in $\S 1$. Characterizations of collectionwise normality and paracompactness in spaces with property $(k)$ are presented in $\S 4$.
\end{abstract}

In 1970, V. Mancuso published a paper [8] which presented the relationships between the notions of mesocompactness and sequential mesocompactness [1], strong and weak cover compactness [5], and Property $Q[12]$.

Theorem 2.4, in [8], seems to establish some of the invariance properties of mesocompact and sequentially mesocompact spaces. There are, however, some errors in the proof of this theorem. With a slight notational change, the proof of the sufficiency of this theorem is correct, for both the compact-finite and the $c s$-finite cases. The error in the proof of the necessity, for the compact-finite case, lies in the fact that (using the notation of [8]) although $f^{-1}(K)$ may meet at most finitely many $U_{\alpha}, f^{-1}(K)$ may meet infinitely many $H_{y}$, because each $U_{\alpha}$ may be used in obtaining infinitely many different $G_{y}$.

Also, the $c s$-finite case does not follow from the compact-finite case, because the inverse image of a covergent sequence, under a perfect mapping need not contain a convergent sequence, which converges to a point in the inverse image of the limit point. An example of this is contained in [3].

In this paper, all spaces will be Hausdorff spaces and all functions will be continuous surjections. By a convergent sequence, we mean the sequence and its limit point.

1. Property $(k)$. A topological space is said to have property $(k)$ 
(property $(\omega)$ [2]), if for each discrete collection of closed sets $\mathscr{F}=\left\{F_{\alpha}: \alpha \in A\right\}$, there exists a compact-finite (cs-finite) collection of open sets $\mathscr{C}=\left\{U_{\alpha}: \alpha \in A\right\}$ such that $F_{\alpha} \subset U_{\alpha}$, for each $\alpha \in A$ and $U_{\alpha} \cap F_{\beta}=\varnothing$ if $\alpha \neq \beta$. Since every compact-finite collection is cs-finite, every space with property $(k)$ has property $(\omega)$. Spaces with property $(\omega)$ were studied and applied to the metrizability of developable spaces in $[2]$.

THEOREM 1. Every mesocompact space has property $(k)$.

Proof. Let $\mathscr{F}=\left\{F_{\alpha}: a \in A\right\}$ be any discrete collection of closed sets in a mesocompact space $X$. For each $\alpha \in A$, let $U_{\alpha}=X-\cup\left\{F_{\beta}\right.$ : $\beta \neq \alpha\}$. Then $\mathscr{U}=\left\{U_{\alpha}: \alpha \in A\right\}$ is an open covering of $X$. Since $X$ is mesocompact, there exists a precise compact-finite open refinement, $\mathscr{V}=\left\{V_{\alpha}: \alpha \in A\right\}$ of $\mathscr{U}$ which covers $X$. The collection $\mathscr{V}$ has the properties required to show $X$ has property $(k)$. This completes the proof.

THEOREM 2. Every collectionwise normal space has property (k).

Proof. If $X$ is collectionwise normal and $\mathscr{F}=\left\{F_{\alpha}: \alpha \in A\right\}$ is a discrete collection of closed sets, then by Dowker's result [6], there exists a discrete collection of open sets $\mathscr{\mathscr { C }}=\left\{U_{\alpha}: \alpha \in A\right\}$ such that $F_{\alpha} \subset U_{\alpha}$, for each $\alpha \in A$. Since every discrete collection is compactfinite, $X$ has property $(k)$.

The proof of Theorem 5 relies on the following mapping theorem spaces with property $(k)$.

THEOREM 3. The perfect image of a space with property (k) has for property $(k)$.

Proof. Let $X$ be a space with property (k), and let $f: X \rightarrow Y$ be a perfect mapping. Let $\mathscr{F}=\left\{F_{\alpha}: \alpha \in A\right\}$ be any discrete collection of closed sets in $Y$. Then $\mathscr{F}^{\prime}=\left\{f^{-1}\left(F_{\alpha}\right): \alpha \in A\right\}$ is a discrete collection of closed sets in $X$. Since $X$ has property $(k)$, there exists a compact-finite collection of open sets $\mathscr{G}=\left\{G_{\alpha}: \alpha \in A\right\}$ such that $f^{-1}\left(F_{\alpha}\right) \subset G_{\alpha}$, for each $\alpha \in A$ and $f^{-1}\left(F_{\alpha}\right) \cap G_{\beta}=\varnothing$, if $\alpha \neq \beta$. Since $f$ is closed, for each $\alpha \in A$ there exists an open set $U_{\alpha} \subset Y$, such that $F_{\alpha} \subset U_{\alpha}$ and $f^{-1}\left(U_{\alpha}\right) \subset G_{\alpha}$. Hence, $F_{\alpha} \cap U_{\beta}=\varnothing$, if $\alpha \neq \beta$. If $K$ is any compact set in $Y$, then $f^{-1}(K)$ is compact in $X$. Thus, since $G_{\alpha} \cap f^{-1}(K) \neq \varnothing$ for at most finitely many $\alpha \in A$, and $f^{-1}\left(U_{\alpha}\right) \subset G_{\alpha}$, $U_{\alpha} \cap K \neq \varnothing$ for at most finitely many $\alpha \in A$. Thus, $\left\{U_{\alpha}: \alpha \in A\right\}$ is compact-finite. Accordingly, $Y$ has property $(k)$. 
2. Characterization of normal mesocompact and normal sequentially mesocompact spaces. The following characterizations are similar to Michael's characterization [9] of the paracompact spaces. That is, the paracompact spaces are precisely the collectionwise normal metacompact spaces. Michael's work, as will be apparent in the proof of Theorem 4, contains all of the ideas and constructions necessary to prove the theorem.

THEOREM 4. A normal space is mesocompact (sequentially mesocompact) iff it is a metacompact space with property $(k)$ (property $(\omega)$ ).

Proof. The necessity is clear. We will prove the sufficiency. Let $X$ be a normal metacompact space with property $(k)$, and let $\mathscr{G}$ be any open covering of $X$. Since $X$ is metacompact, there exists a point-finite open refinement $\mathscr{C}$ of $\mathscr{C}$. (To avoid confusion, Michael's notation will be used in this proof.) By Michael's construction (in the proof of his Theorem 2 [9] and the hypothesis that $X$ has property $(k)$ (property $(\omega)$ ), there exists a sequence $\left\{\mathscr{W}_{i}: i=0,1,2, \cdots\right\}$ of collections of open subsets of $X$ such that, the following conditions are satisfied for each $i$ :

(a) Every $W \in \mathscr{W}_{i}$ is a subset of some $U \in \mathscr{W}$.

(b) $\mathscr{W}_{i}$ is compact-finite (cs-finite).

(c) If $x \in X$ is an element of at most $i$ elements of $\mathscr{W}$, then there exists $W \in \mathscr{W}_{n}, 0 \leqq n \leqq i$, such that $x \in W$.

(d) Every $x \in \cup\left\{W: W \in \mathscr{W}_{i}\right\}$ is an element of at least $i$ elements of $\mathscr{U}$.

For each $i=0,1,2, \cdots$, let $W_{i}=U\left\{W: W \in \mathscr{W}_{i}\right\} . \quad$ By (c) and (d), $\left\{W_{i}\right\}$ is a point-finite open covering of $X$. Morita [10, Theorem 3 and Lemma 3] has shown that, every countable point-finite covering of a normal space has a locally finite refinement. Accordingly, since $X$ is normal, $\left\{W_{i}\right\}$ has a locally finite open refinement which covers $X,\left\{V_{i}\right\}$, where $V_{i} \subset W_{i}$, for each $i$. By (a), the collection $\mathscr{H}=$ $\bigcup_{i=0}^{\infty}\left\{V_{i} \cap W: W \in \mathscr{W}_{i}\right\}$ is an open refinement of $\mathscr{U}$, and thus of $\mathscr{G}$. Since locally finite families are compact-finite (cs-finite), for each compact set $K \subset K$ (convergent sequence $K$ in $X$ ), $V_{i} \cap K \neq \varnothing$, for at most finitely many $i$. Further, since $\mathscr{W}_{i}$ is compact-finite (csfinite), for each $i$, by (b), $\left(V_{i} \cap W\right) \cap K \neq \varnothing$ for at most finitely many sets in $\mathscr{H}$. Accordingly, $\mathscr{H}$ is a compact-finite (cs-finite) open refinement of $\mathscr{G}$ which covers $X$, and $X$ is mesocompact (sequentially mesocompact).

An example of a Tychonoff sequentially mesocompact space which is not mesocompact is presented in [4, Example 2.1]. This answers one of the open questions in Remark 1.13 [8]. 
3. Mapping theorems. The following theorems use an important theorem of Worrell [11], which states that the closed image of a metacompact space is metacompact.

THEOREM 5. The perfect image of a normal mesocompact space is a normal mesocompact space.

Proof. Let $X$ be a normal mesocompact space, and let $f: X \rightarrow Y$ be a perfect mapping. Since perfect mappings are closed, $Y$ is metacompact, by Worrell's theorem. Clearly, $Y$ is normal. Since $f$ is perfect, $Y$ has property, $(k)$, by Theorem 3. Hence, by Theorem 4, $Y$ is a normal mesocompact space. This completes the proof.

As was noted before, the perfect mappings do not satisfy the sequential conditions necessary for the mapping theorem, for the normal sequentially mesocompact sepaces, to follow as a consequence of Theorem 5. Some of the notions introduced by Franklin [7] will be used to define a mapping with the desired properties. Franklin calls a set, $H$, sequentially closed, if no sequence in $H$ converges to a point not in $H$, and a space sequential, if every sequentially closed set is closed. We will call a mapping, $f: X \rightarrow Y$, presequential, if for each convergent sequence $\left\{p_{i}\right\}$ in $Y, p_{i} \rightarrow p$, which is not eventually equal to $p, \cup\left\{f^{-1}\left(p_{i}\right): i \in N, p_{i} \neq p\right\}$ is not sequentially closed. Although the perfect mappings need not be presequential [3], many mappings of interest are presequential. For example, the countable-to-one perfect mappings and the quotient mappings defined on sequential spaces are presequential. That is, any quotient map such that, the preimage of a convergent sequence is sequential as a subspace, is presequential.

Theorem 6. If $f: X \rightarrow Y$ is a presequential mapping, then for each convergent sequence $\left\{p_{i}\right\}$ in $Y, p_{i} \rightarrow p$, there exists a convergent sequence $\left\{q_{n}\right\}$ in $X$ such that, $q_{n} \in f^{-1}\left(p_{i_{n}}\right)$, for some subsequence $\left\{p_{i_{n}}\right\}$, and $\left\{q_{n}\right\}$ converges to some $q \in f^{-1}(p)$.

Proof. The proof of Theorem B in [3], contains the proof of this theorem.

THEOREM 7. The closed presequential image of a space with property $(\omega)$ has property $(\omega)$.

Proof. Let $X$ be a space with property $(\omega)$, and let $f: X \rightarrow Y$ be a closed presequential map. Let $\mathscr{F}=\left\{F_{\alpha}: \alpha \in A\right\}$ be any discrete collection of closed subsets of $Y$. Then $\mathscr{F}^{\prime}=\left\{f^{-1}\left(F_{\alpha}\right): \alpha \in A\right\}$ is a discrete closed collection in $X$. Since $X$ has property $(\omega)$, there exists 
a $c s$-finite collection of open sets $\mathscr{G}=\left\{G_{\alpha}: \alpha \in A\right\}$ such that $f^{-1}\left(F_{\alpha}\right) \subset G_{\alpha}$, for each $\alpha \in A$ and $f^{-1}\left(F_{\alpha}\right) \cap G_{\beta}=\varnothing$, if $\alpha \neq \beta$. Since $f$ is closed, for each $\alpha \in A$ there exists an open set in $Y, U_{\alpha}$, such that $F_{\alpha} \subset U_{\alpha}$ and $f^{-1}\left(U_{\alpha}\right) \subset G_{\alpha}$. Hence $F_{\alpha} \cap U_{\beta}=\varnothing$, if $\alpha \neq \beta$.

The collection $\mathscr{\mathscr { C }}=\left\{U_{\alpha}: \alpha \in A\right\}$ is point-finite in $Y$; because, if any point $p \in Y$ were an element of infinitely many sets, $U_{\alpha}$, then any point $q \in f^{-1}(p)$ would be an element of infinitely many sets, $f^{-1}\left(U_{\alpha}\right)$, and thus of infinitely many sets, $G_{\alpha}$. Thus contradicting the csfiniteness of $\mathscr{G}$.

Assume $\mathscr{U}$ is not $c s$-finite. Then there is some convergent sequence $\left\{p_{i}\right\}$ in $Y, p_{i} \rightarrow p$, such that $U_{\alpha} \cap\left(\left\{p_{i}: i \in N\right) \cup\{p\}\right) \neq \varnothing$, for infinitely many $\alpha \in A$. By the point-finiteness of $\mathscr{C}$, there is a subsequence $\left\{p_{i_{j}}\right\}$ of $\left\{p_{i}\right\}$ such that $p_{i_{j}} \in U_{\alpha(j)} \in \mathscr{U}$ and $U_{\alpha(j)} \neq U_{\alpha(k)}$, for $j \neq k$. Since $p_{i_{j}} \rightarrow p$ and $\mathscr{U}$ is not $c s$-finite on $\left\{p_{i_{j}}\right\}$, for simplicity we can let the subsequence be $\left\{p_{i}\right\}$ itself. Since $f$ is presequential, there exists a convergent sequence $\left\{q_{n}\right\}$ in $X$ such that, $q_{n} \in f^{-1}\left(p_{i_{n}}\right)$, for some subsequence $\left\{p_{i_{n}}\right\}$, and $\left\{q_{n}\right\}$ converges to some $q \in f^{-1}(p)$. Thus, $q_{n} \in f^{-1}\left(U_{\alpha\left(i_{n}\right)}\right)$, for each $n \in N$. Since $U_{\alpha\left(i_{n}\right)} \neq U_{\alpha\left(i_{m}\right)}$, if $n \neq m$ implies $f^{-1}\left(U_{\alpha\left(i_{n}\right)}\right) \neq f^{-1}\left(U_{\alpha\left(i_{m}\right)}\right), f^{-1}\left(U_{\alpha}\right) \cap\left(\left\{q_{n}: n \in N\right\} \cup\{q\}\right) \neq \varnothing$, for infinitely many $\alpha \in A$. Since $f^{-1}\left(U_{\alpha}\right) \subset G_{\alpha}$, for each $\alpha \in A, G_{\alpha} \cap\left(\left\{q_{n}\right.\right.$ : $n \in N\} \cup\{q\}) \neq \varnothing$, for infinitely many $\alpha \in A$. But this is a contradiction to the $c s$-finiteness of $\mathscr{G}$. Hence $\mathscr{C}$ is $c s$-finite. Accordingly, $Y$ has property $(\omega)$ and this completes the proof.

By Worrell's Theorem [11], the invariance of normality under closed maps, Theorem 7 and finally Theorem 4, the following theorem is established.

THEOREM 8. The closed presequential image of a normal sequentially mesocompact space is a normal sequentially mesocompact space.

Example 2.2 in [4] establishes that the perfect image of a Tychonoff sequentially mesocompact space need not be sequentially mesocompact.

4. Collectionwise normality and paracompactness of spaces with property $(k)$. The following theorem, which is analogous to [2, Corollary 3.2], is a consequence of the characterization of collectionwise normal spaces, presented in [2, Theorem 3.1].

THEOREM 9. A k-space is collectionwise normal iff it is a normal space with property $(k)$.

Accordingly from Michael's theorem [9] and Theorem 6, or from 
Theorem 4 and [1, Theorem 3.2], paracompactness in $k$-spaces is characterized in the following theorem.

THEOREM 10. A k-space is paracompact iff is a normal metacompact space with property $(k)$.

The collectionwise normality and paracompactness of spaces with property $(\omega)$ is presented in [2].

\section{REFERENCES}

1. J. R. Boone, Some characterizations of paracompactness in k-spaces, Fund. Math., 72 (1971), 146-154.

2. —_, A metrization theorem for developable spaces, Fund. Math., 73 (1971), 79-83.

3. - Nearness preserving conditions for inverse images, Notices Amer. Math., Soc., 18 (1971), 1112.

4. - Examples relating to mesocompact and sequentially mesocompact spaces, Fund. Math., 77 (1972), 91-93.

5. R. C. Briggs, III, A comparison of covering properties $T_{3}$ and in $T_{4}$ spaces, Doctoral Dissertation, Univ. of Houston, 1968.

6. C. H. Dowker, On a theorem of Hanner, Ark. Mat., 2 (1952), 307-313.

7. S. P. Franklin, Spaces in which sequences suffice, Fund. Math., 57 (1965), 107-115.

8. V. J. Mancuso, Mesocompactness and related properties, Pacific J. Math., 33 (1970), $345-355$.

9. E. Michael, Point-finite and locally finite coverings, Canad. J. Math., 7 (1955), 275-279. 10. K. Morita, Star-finite coverings and the star-finite property, Math. Japonicae, 1 (1948), 60-68.

11. J. M. Worrell, Jr., The closed continuous images of metacompact spaces, Port. Math., 25 (1966), 175-179.

12. J. N. Younglove, Concerning dense metric subspaces of certain non-metric spaces, Fund. Math., 48 (1960), 15-25.

Received September 14, 1971.

TEXAS A \& M UNIVERSITY 


\section{PACIFIC JOURNAL OF MATHEMATICS}

\section{EDITORS}

H. SAMELSON

Stanford University

Stanford, California 94305

C. R. Hовву

University of Washington

Seattle, Washington 98105
J. DugundJI

Department of Mathematics University of Southern California

Los Angeles, California 90007

RICHARD ARENS

University of California

Los Angeles, California 90024

\section{ASSOCIATE EDITORS}

E. F. BECKENBACH

B. H. NeumanN

F. WOLF

K. YoSHIDA

\section{SUPPORTING INSTITUTIONS}

UNIVERSITY OF BRITISH COLUMBIA

CALIFORNIA INSTITUTE OF TECHNOLOGY

UNIVERSITY OF CALIFORNIA

MONTANA STATE UNIVERSITY

UNIVERSITY OF NEVADA

NEW MEXICO STATE UNIVERSITY

OREGON STATE UNIVERSITY

UNIVERSITY OF OREGON

OSAKA UNIVERSITY
UNIVERSITY OF SOUTHERN CALIFORNIA

STANFORD UNIVERSITY

UNIVERSITY OF TOKYO

UNIVERSITY OF UTAH

WASHINGTON STATE UNIVERSITY

UNIVERSITY OF WASHINGTON

AMERICAN MATHEMATICAL SOCIETY

NAVAL WEAPONS CENTER

Printed in Japan by International Academic Printing Co., Ltd., Tokyo, Japan 


\section{Pacific Journal of Mathematics}

\section{Vol. 44, No. $1 \quad$ May, 1973}

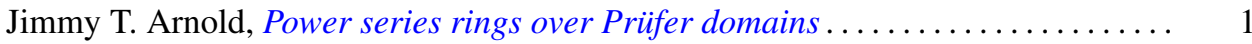

Maynard G. Arsove, On the behavior of Pincherle basis functions . . . . . . . . . 13

Jan William Auer, Fiber integration in smooth bundles ................. 33

George Bachman, Edward Beckenstein and Lawrence Narici, Function algebras

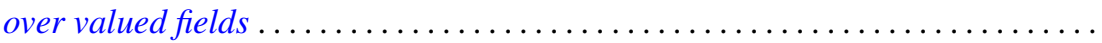

Gerald A. Beer, The index of convexity and the visibility function . . . . . . . . . . .

James Robert Boone, A note on mesocompact and sequentially mesocompact

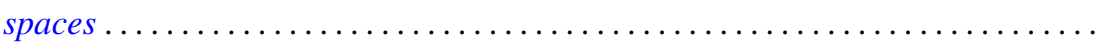

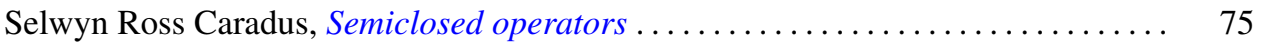

John H. E. Cohn, Two primary factor inequalities . . . . . . . . . . . . . . . 81

Mani Gagrat and Somashekhar Amrith Naimpally, Proximity approach to

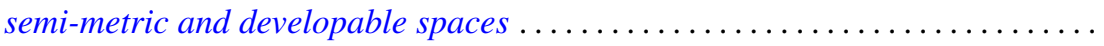

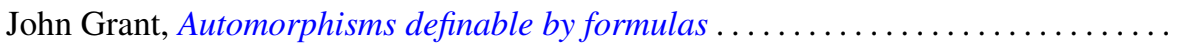

Walter Kurt Hayman, Differential inequalities and local valency ..............

Wolfgang H. Heil, Testing 3-manifolds for projective planes . . . . . . . . . . . . .

107

Melvin Hochster and Louis Jackson Ratliff, Jr., Five theorems on Macaulay

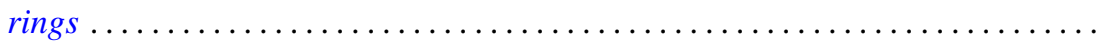

Thomas Benton Hoover, Operator algebras with reducing invariant subspaces ....

James Edgar Keesling, Topological groups whose underlying spaces are separable

Fréchet manifolds...

Frank Leroy Knowles, Idempotents in the boundary of a Lie group . .

191

George Edward Lang, The evaluation map and EHP sequences ...

201

Everette Lee May, Jr, Localizing the spectrum . . . . . . . . . . . .

211

Frank Belsley Miles, Existence of special $K$-sets in certain locally compact abelian groups.

Susan Montgomery, A generalization of a theorem of Jacobson. II . .

T. S. Motzkin and J. L. Walsh, Equilibrium of inverse-distance forces in

three-dimensions.

Arunava Mukherjea and Nicolas A. Tserpes, Invariant measures and the converse

of Haar's theorem on semitopological semigroups .

James Waring Noonan, On close-to-convex functions of order $\beta$

Donald Steven Passman, The Jacobian of a growth transformation

Dean Blackburn Priest, A mean Stieltjes type integral ........ .

Joe Bill Rhodes, Decomposition of semilattices with applications to topological

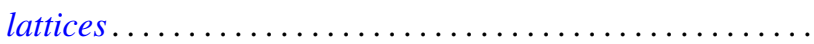

Claus M. Ringel, Socle conditions for $\mathrm{QF}-1$ rings ..........

Richard Rochberg, Linear maps of the disk algebra

Roy W. Ryden, Groups of arithmetic functions under Dirichlet convolution . .

Michael J. Sharpe, A class of operators on excessive functions

Erling Stormer, Automorphisms and equivalence in von Neumann algebras ..

Philip C. Tonne, Matrix representations for linear transformations on series

analytic in the unit disc. 